\title{
The Monocarboxylate Transporter Inhibitor $\alpha$-Cyano-4-Hydroxycinnamic Acid Disrupts Rat Lung Branching
}

\author{
Sara Granja ${ }^{a, b}$ Filipa Morais-Santos ${ }^{a, b}$ Vera Miranda-Gonçalves ${ }^{a, b}$ \\ Manuel Viana-Ferreira ${ }^{a, b, c}$ Rosete Nogueira ${ }^{a, b, d} \quad$ Cristina Nogueira-Silva ${ }^{a, b, e}$ \\ Jorge Correia-Pinto ${ }^{a, b, f}$ Fátima Baltazar ${ }^{a, b}$ \\ aLife and Health Sciences Research Institute (ICVS), School of Health Sciences, University of Minho, \\ Braga, ${ }^{b} I C V S / 3 B$ 's-PT Government Associate Laboratory, Braga/Guimarães, 'Department of General \\ Surgery, ULSAM, Hospital of Viana do Castelo, Viana do Castelo, ${ }^{\mathrm{D} C G C}$ Genetics/Centro de Genética \\ Clínica - Laboratory of Pathology, Porto, eDepartment of Obstetrics and Gynecology, Hospital of Braga,

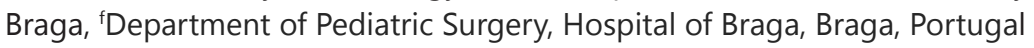

\section{Key Words}

Monocarboxylate transporters $\cdot$ Lung morphogenesis $\cdot$ Cell metabolism $•$ Embryo development

\begin{abstract}
Background/Aims: The human embryo develops in a hypoxic environment. In this way, cells have to rely on the glycolytic pathway for energy supply, leading to an intracellular accumulation of monocarboxylates such as lactate and pyruvate. These acids have an important role in cell metabolism and their rapid transport across the plasma membrane is crucial for the maintenance of intracellular $\mathrm{pH}$ homeostasis. This transport is mediated by a family of transporters, designated by monocarboxylate transporters (MCTs), namely isoforms 1 and 4. MCT1/4 expression is regulated by the ancillary protein CD147.The general aim of this study was to characterize the expression pattern of MCT1/4, CD147 and the glucose transporter GLUT1 during human fetal lung development and elucidate the role of MCTs in lung development. Methods: The expression pattern of MCT1/4 and GLUT1 was characterized by immunohistochemistry and fetal lung viability and branching were evaluated by exposing rat fetal lung explants to $\mathrm{CHC}$, an inhibitor of MCT activity. Results: Our findings show that all the biomarkers are differently expressed during fetal lung development and that $\mathrm{CHC}$ appears to have an inhibitory effect on lung branching and viability, in a dose dependent way. Conclusion: We provide evidence for the role of MCTs in embryo lung development, however to prove the dependence of MCT activity further studies are waranted.
\end{abstract}




\section{Introduction}

Oxygen levels are a metabolic regulator in the balance between oxidative phosphorylation and glycolysis. In the presence of oxygen, normal cells rely on oxidative phosphorylation for ATP generation however, when exposed to low levels of oxygen they shift to glycolysis [1, 2]. Increased glycolytic metabolism generates intracellular acids, leading to a decrease in intracellular $\mathrm{pH}$ (pHi). These acids have an important role in cell metabolism and their rapid transport across the plasma membrane is crucial for pHi homeostasis. This transport could be carried out by a family of transporters, designated as monocarboxylate transporters (MCTs) [3].

MCTs are encoded by the family of genes SLC16A, and based on sequence homologies, 14 members of MCT family were identified [4], although only MCT1-4 are able to perform proton-linked monocarboxylate transport [4-6]. MCT1 and MCT4 are proton symporters, which have lactate as common substrate and each isoform exhibits different biochemical properties, such as substrate specificity and inhibitor sensitivity [4]. MCT1 is the most well-studied and functionally characterized member of the MCT family and has the widest tissue distribution $[4,6]$ while MCT4 is a transporter of low affinity mostly associated with the export of lactate from cells with high glycolytic rates [7-9]. There are a variety of MCT inhibitors described in the literature, such as $\alpha$-cyano-4-hydroxycinnamate (CHC), which has been widely shown to inhibit MCTs, exhibiting different affinities for each isoform $[4,10]$. Substrate affinity has been associated with the specific distribution of each transporter in the tissues, depending on their metabolic activity. Ancillary proteins are required to maintain the catalytic activity of MCTs, as well as for their translocation to the plasma membrane. MCT1 and MCT4 require a protein named CD147 [11-13].

Localized hypoxia is a normal feature of embryo development [14], and this low fetal oxygen environment is one of the most important extracellular factors for lung morphogenesis $[15,16]$, which is a complex process $[17,18]$. Studies have shown that embryonic and fetal development are influenced by oxygen availability [19] and may, at some stage, rely primarily on glycolytic metabolism for energy production [20,21]. Some studies have already demonstrated the expression of MCTs in pre-implantation of the embryo [22-24], during early embryonic development of human and mouse [7], in the developing heart of chick embryos [21] and in the forebrain of rat embryos [25]. However, the role of hypoxia during embryo/fetal development is not clearly understood. Some studies show that HIF1 plays a major role in mediating pulmonary vascular remodelling in response to hypoxia $[26,27]$ while others suggest that severe hypoxia in later gestation can cause damage [17, 28]. Troug et al. [17] observed that chronic hypoxia at the end of gestation interrupted lung development and they detected upregulation of MCT4 in these tissues. However, there are no studies demonstrating the expression of MCTs during the process of embryogenesis and specifically lung morphogenesis. In this work, we evaluate for the first time the expression of MCT1/4, CD147, the glucose transporter 1 (GLUT1) and the biomarker of hypoxia carbonic anhydrase 9 (CAIX) in a human fetal lung series and evaluate the effect of MCT inhibition on viability and branching morphogenesis of rat fetal lungs.

\section{Materials and Methods}

Human tissue specimens

All the cases were retrieved from the laboratory of pathology from CGC genetics in Porto. Fetal human tissues were selected from late spontaneous abortions between $12^{\text {th }}$ and $37^{\text {th }}$ weeks of gestational age. Spontaneous abortion occurred in all cases, including one case of fetal focal defects of the distal extremities in a context of amniotic bands, and other case with clinical features suggestive of chromosomal anomalies confirmed by cytogenetic studies (trisomy 21), which had a cardiac ventricular septal defect. None of the other fetus had developmental abnormalities. All cases had permission for autopsy and were donated for 


\begin{tabular}{|c|c|c|}
\hline Cellular Physiolosy & Cell Physiol Biochem 2013;32:1845-1856 & \\
\hline and Biochemistry & $\begin{array}{l}\text { DOI: } 10.1159 / 000356617 \\
\text { Published onlIne: December 20, } 2013\end{array}$ & $\begin{array}{l}\text { (c) } 2013 \text { S. Karger AG, Basel } \\
\text { www.karger.com/cpb }\end{array}$ \\
\hline
\end{tabular}

pathologic/scientific research. All tissues were collected after informed consent in accordance with the Ethics Guidelines of CGC clinics.

\section{Animal model}

Animal experiments were performed according to the Portuguese law for animal welfare. Animals were housed in an accredited rat house and treated as specified in the Guide for the Care and Use of Laboratory Animals published by the US National Institutes of Health (National Institutes of Health Publication No.85-23, revised 1996). Sprague-Dawley female rats (Charles-River, Barcelona, Spain) were maintained in appropriate cages under controlled conditions, fed with commercial solid food. After mating, they were checked for intracoital plugging and all pregnant rats were sacrificed by decapitation. Fetal rat lung tissues were obtained from fetuses harvested by caesarean section at 15.5 (pseudoglandular phase), 17.5 (canalicular phase), 19.5 and 21.5 (saccular phase) day post-conception (dpc). Samples were fixed in formalin, embedded in paraffin and $4 \mu \mathrm{m}$ sections were used for immunohistochemistry (IHC).

\section{Immunohistochemistry}

IHC for MCT1 (1:200 dilution; AB3538P; Chemicon International) was performed according to the avidin-biotin-peroxidase principle (R.T.U. Vectastin Elite ABC kit; Vector Laboratories), as previously described by our group [29]. MCT4 (1:500 dilution, H-90; sc-50329; Santa Cruz Biotechnology), GLUT1 (1:500 dilution; ab15309; Abcam) and CAIX (1:1000 dilution; ab15086; Abcam) IHC was performed with the Ultravision Detection System Anti-polyvalent, HRP (Lab Vision Corporation), as previously described [29, 30]. CD147 (1:300 dilution; sc-71038; Santa Cruz Biotechnology diluted) IHC was performed using the UltraVision ONE Detection System: HRP Polymer (Lab Vision Corporation). In brief, paraffin embedded sections were deparaffinized in xylene and hydrated in a graded series of ethanol solutions. For antigen retrieval, slides were incubated with $1 \mathrm{mM}$ EDTA buffered solution (pH 8.0) for $20 \mathrm{~min}$ in water bath at $98^{\circ} \mathrm{C}$. Slides were incubated overnight at room temperature with the primary antibody CD147. Tissues were stained with 3,3'-diamino-benzidine (DAB + Substrate System, DakoCytomation) for $10 \mathrm{~min}$ and counterstained with haematoxylin. Colon carcinoma tissue was used as positive control for MCT1, MCT4, CD147 and GLUT1 and normal stomach tissue for CAIX. Stained slides were evaluated and then photographed under a bright field microscope Olympus BX61.

\section{Immunofluorescence (IF)}

Double staining for MCT1, MCT4, CD147, GLUT1 or CAIX with cell specific markers for mesenchymal cells (Vimentin) and epithelial cells (E-cadherin) was performed by IF. Sections were incubated for each protein, MCT1 (1:200 dilution, O.N.; AB3538P; Chemicon International), MCT4 (1:500 dilution, 2h; H-90; sc50329; Santa Cruz Biotechnology), CD147 (1:750 dilution, 2h; 18-7344, ZYMED Laboratories Inc.), GLUT1 (1:500 dilution, 2h; ab15309; Abcam) and CAIX (1:1000 dilution, 2h; ab15086; Abcam) and incubated with monoclonal mouse anti-Vimentin (1:150, $30 \mathrm{~min}$; M0725; DAKO), or with monoclonal mouse antiE-cadherin (1/100, O.N; sc-21791; Santa Cruz Biotechnology). After rinsing in PBS/0.1\% Tween, sections were incubated with an anti-mouse secondary antibody labelling in red (Alexa fluor 594; 1:250, 1h; A11032; Invitrogen) or anti-rabbit antibody labelling in green (Alexa fluor 488; 1:500, 1h; A11008; Invitrogen). Nuclei were counterstained with 4',6-diamidino-2-phenylindole (dapi) and sections were mounted in Vectashield Mounting Media (Vector Laboratories). Stained slides were photographed with a fluorescence microscope (Olympus BX61).

\section{Rat fetal lung explant cultures}

Fetuses were removed by caesarean section at $13.5 \mathrm{dpc}$. Harvesting and dissection of the lungs was made in Dulbecco's Phosphate Buffered Saline (DPBS - Lonza) under a dissection microscope (Leica MZFLIII, Wetzlar, Germany). Lungs were transferred to Nucleopore membranes with $8 \mu \mathrm{m}$ pore size (Whatman, Clifton, NJ, USA) and incubated in a 24-well culture plates from Costar (Corning, NY, USA). Membranes were pre-soaked in DMEM (Lonza) for 1 hour before the explants were placed on them. Floating cultures of the explants were incubated in $200 \mu \mathrm{l}$ of 50\% Dulbecco's modified eagle medium (DMEM), 50\% nutrient mixture F-12 (Invitrogen, Carlsbad, CA, USA) supplemented with $100 \mathrm{mg} / \mathrm{ml}$ streptomycin, 100 units/ $\mathrm{mL}$ penicillin (Invitrogen), $0.25 \mathrm{mg} / \mathrm{mL}$ ascorbic acid (Sigma-Aldrich, St Louis, MO, USA) and 10\% fetal calf serum (Invitrogen). After 1 hour incubation, $200 \mu \mathrm{l}$ of medium with the inhibitor (CHC) at pH 7.4 was 
added. Fetal lung explants were incubated for 96 hours, at $37^{\circ} \mathrm{C}$ and $5 \% \mathrm{CO}_{2}$. The medium with inhibitor was replaced every $24 \mathrm{~h}$ for 4 days. Fetal lungs were photographed daily and D0 was considered the first day and D4 the last. At the end of the incubation time, explants were washed in PBS and stored at $-80^{\circ} \mathrm{C}$ until use.

\section{Morphometric analysis of lung explants}

Branching morphogenesis was monitored daily by photographing the explants. At day 0 (D0: $0 \mathrm{~h}$ ) and 4 (D4: 96h) of culture, the total number of peripheral airway buds (branching), the epithelial perimeter and total explant area in all lung explants were measured using a specific software developed by the laboratory (unpublished data). For all experimental conditions, results of branching and epithelial perimeter were expressed as D4/D0 ratio.

\section{Western blot}

Expression of MCT1 and MCT4 was also evaluated by Western blotting in rat embryo samples. Total protein was extracted from the lung explants at stages 16, 18, 20 and 22. After maceration of the lungs with lysis buffer, the homogenate was centrifuged at $13000 \mathrm{rpm}$ for $15 \mathrm{~min}$ at $4^{\circ} \mathrm{C}$. The supernatants were collected and protein concentrations were determined by the DC Protein Assay Kit (500-0113, BioRad). $40 \mu \mathrm{g}$ of protein of each sample were separated on $10 \%(\mathrm{w} / \mathrm{v})$ polyacrylamide gels and transferred onto nitrocellulose membranes (Amersham Biosciences). After that, membranes were blocked with $0.1 \%$ Tween, $1 \%$ BSA in TBS for $1 \mathrm{~h}$ and then incubated overnight at $4^{\circ} \mathrm{C}$ with the primary polyclonal antibodies for MCT1 (1:200 dilution; AB3538P; Chemicon International) and MCT4 (1:1000 dilution, H-90; sc-50329; Santa Cruz Biotechnology). After washing with TBS-0.1\%Tween, membranes were incubated with an anti-rabbit secondary antibody coupled to horseradish peroxidase (SantaCruz Biotechnology, 1:5000 in TBS-0.1\% Tween, 1\% BSA). At the end, bound antibodies were visualized by chemiluminescence (Supersignal West Femto kit; Pierce). For loading control, blots were probed with $\beta$-tubulin monoclonal antibody (1:200000 dilution, Abcam, UK).

\section{Results}

\section{Expression of MCTs along human lung embryo development}

We evaluated the expression of MCT1, MCT4, CD147, CAIX and GLUT1 in human fetal lung samples (Fig. 1). Lung tissue samples were collected and divided into 4 groups, according to the lung development phases: pseudoglandular (11-17 weeks of gestation; $\mathrm{n}=7)$; canalicular (18-25 weeks; $\mathrm{n}=8)$; saccular (24-36 weeks; $\mathrm{n}=5)$ and alveolar $(37-40$ weeks; $n=3$ ).

All proteins were expressed in the pseudoglandular phase of human lung gestation. MCT1 immunoreactivity was primarily detected in mesenchymal cells and in primitive epithelial alveolar cells (Fig. 1A). From 15 weeks of gestation, staining decreased in mesenchymal cells and started to appear also in the bronchial epithelium. In contrast, MCT4 and GLUT1 proteins were consistently early detected in bronchial and primitive alveolar epithelial cells and absent in mesenchymal cells. CD147 expression was only observed from week 16 in mesenchymal cells, primitive epithelial alveolar cells and on the bronchial epithelial cells, however staining was weak. At 17 weeks of gestation, CD147 was strongly detected in the basal region of the primitive epithelial alveolar cells and also on bronchial epithelium and mesenchymal cells. CAIX expression was restricted to the bronchial epithelial cells along this phase.

During the canalicular phase, MCT1 expression continues to decrease in mesenchymal cells and to become more intense in the apical region of bronchial epithelium cells and in the basal region of alveolar cells. MCT4 and GLUT1 have similar expression patterns, maintaining the expression in the bronchial epithelial cells from 18 to 25 weeks of gestation and becoming absent in alveolar cells from week 19. MCT4 was focally detected in the glands of the bronchial epithelium. CD147 and CAIX expression varies during this phase. First, CD147 is only detected in the epithelium of the principal bronchus while CAIX is absent. 


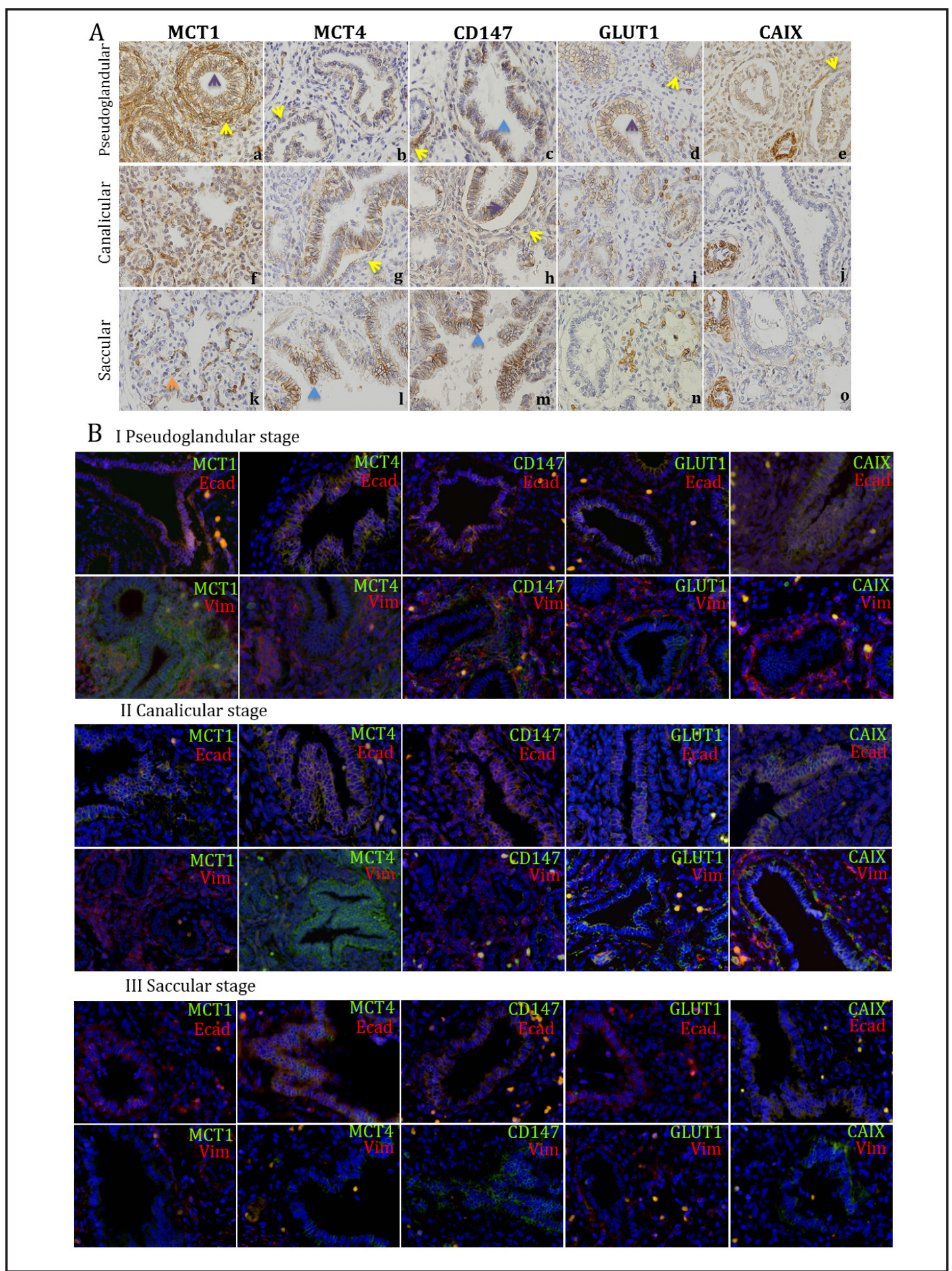

Fig. 1. A - Immunohistochemical analysis of MCT1 (a,f,k), MCT4 (b,g,l), CD147 (c,h,m), GLUT1 (d,I,n) and CAIX $(e, j, o)$ during human fetal lung development. Immunoreaction was differently detected in mesenchymal cells (yellow arrow), primitive alveolar cells (purple arrow), bronchial epithelium (blue arrow) and alveolar type II cells (orange arrow). B- Immunofluorescence staining of MCT1, MCT4, CD147, GLUT1 and CAIX co-localized with mesenchymal cell type marker, Vimentin, and with epithelial marker E-cadherin in I: pseudoglandular stage, II: Canalicular stage and III: Saccular stage. Vim (Vimentin) in red, Ecad (E-cadherin) in red and MCT1, MCT4, CD147, GLUT1 and CAIX in green. Counterstained with dapi (blue). Original magnification $\times 400$. 


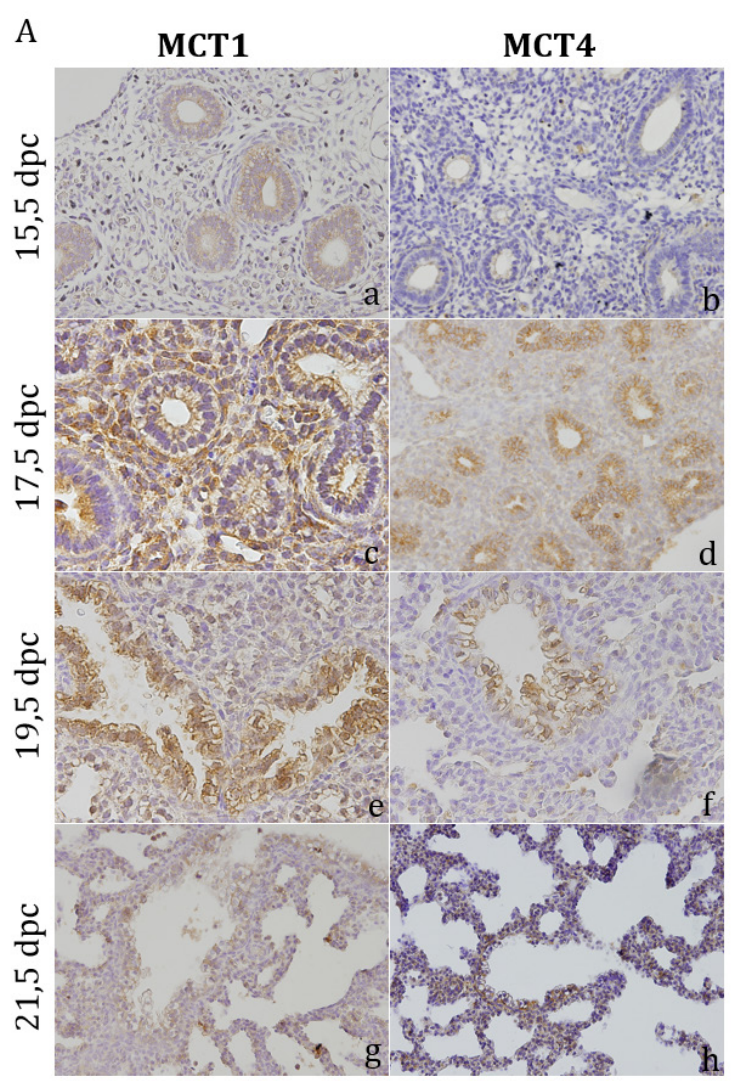

B

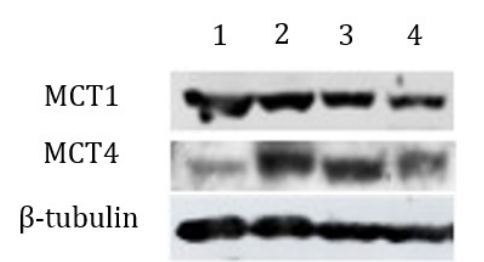

Fig. 2. A - Expression pattern of MCT1 (left column) and MCT4 (right column) during rat fetal lung development. Both isoforms are expressed throughout all stages of lung development studied, from early $15.5 \mathrm{dpc}$ until late $21.5 \mathrm{dpc}$. Original magnification $\times 200 \mathrm{a}, \mathrm{b}, \mathrm{d}, \mathrm{g}, \mathrm{h})$ and $\mathrm{x} 400$ (c,e,f). B - Western blot analysis of both MCT1 and MCT4 during the lung development phases: (1) 15.5 dpc; (2) 17.5 dcp; (3) 19.5 dpc; (4) 21.5 dpc. $\beta$-tubulin was used as loading control.

From week 22, CD147 is expressed in mesenchymal cells, and both CD147 and CAIX are present in epithelial alveolar and bronchial cells.

In saccular/alveolar phase, MCT1 expression decreased in the bronchial epithelium and is detected in pneumocytes type 2. MCT4 expression remained similar to canalicular phase while GLUT1 is absent. CD147 immunoreactivity is detected predominantly in the bronchi epithelium, being weak or even absent in the alveolar epithelium. CAIX expression is detected in both alveolar and bronchial epithelium.

By IF staining, we confirmed the cell co-localization of the proteins described above, with cell specific markers, vimentin for mesenchymal cells and E-cadherin for epithelial cells. As observed in Figure 1B, MCT1 and CD147 co-localized with vimentin; while the other proteins co-localized only with E-cadherin at the pseudoglandular stage (Fig. 1BI). In the canalicular (Fig. 1BII) and saccular (Fig. 1BIII) stage, all proteins were mainly localized in the epithelial cells.

CD147, CAIX and MCT1 were also detected in the mesothelium. CAIX is mainly expressed in vessels during all stages of gestation.

\section{Expression of MCTs in fetal rat lung tissues}

Firstly we confirmed the expression of MCTs in rat fetal lung tissues. Thus, we performed IHC on samples corresponding to pseudoglandular (15.5 dpc), canalicular (17,5 
Fig. 3. A - Branching morphogenesis in rat lung explants treated with $\mathrm{CHC}(0.5 \mathrm{mM}$ and $1 \mathrm{mM}$ ). The first line represents lung explants at D0, the second line represent lung explants treated with different doses of CHC at D4. ( $\mathrm{n}=12$ per condition); B Morphometric analysis; i/ii: epithelial external and internal perimeter; iii: total number of peripheral airway buds; iv/v epithelial area. Numeric results are expressed as D4/D0 ratio. $\mathrm{p}<0.05$; * vs control (DMSO).

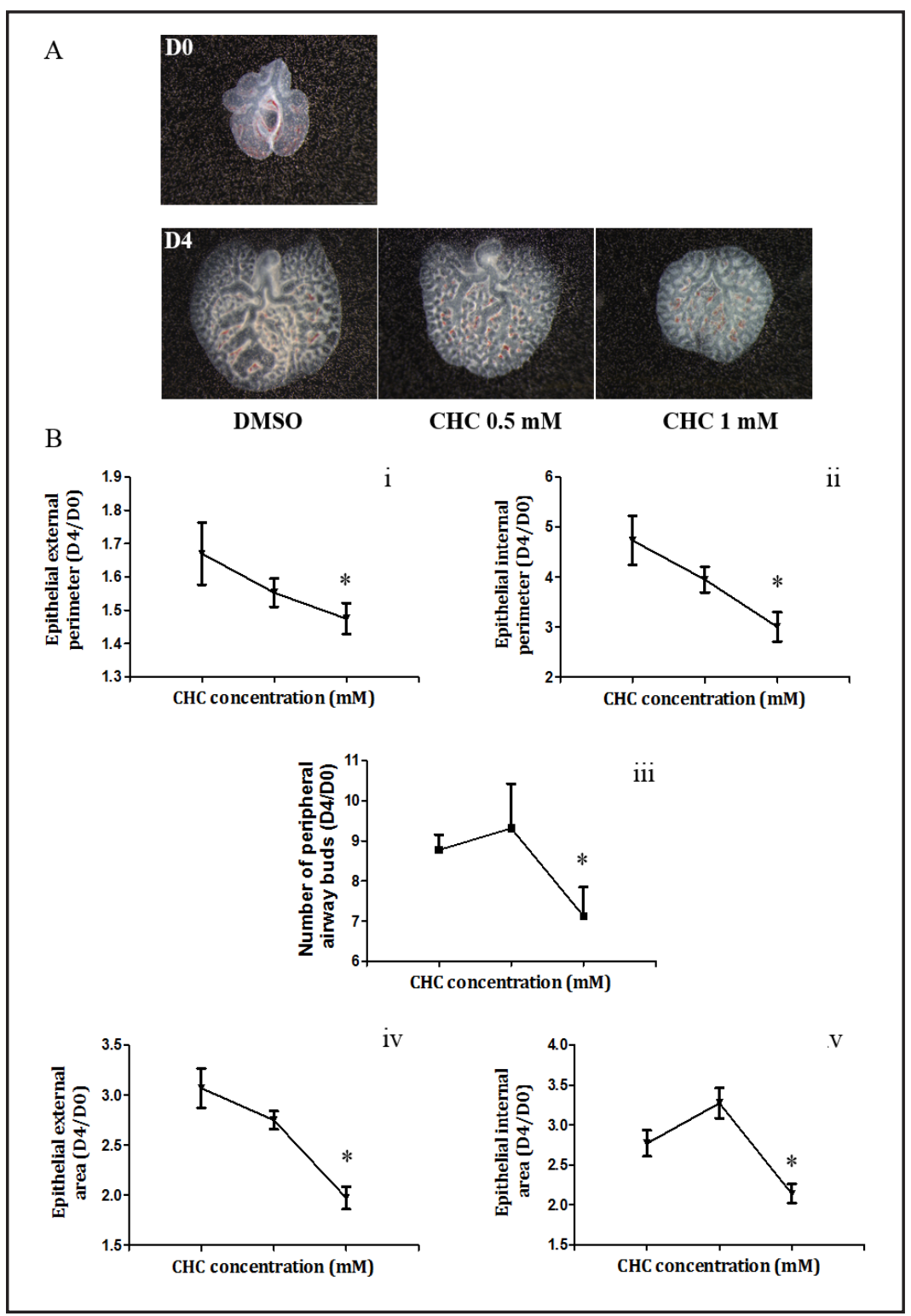

dpc), saccular (19.5 dpc) and alveolar phase (21.5 dpc). By analysis of Fig. 2A, it is possible to see that both isoforms are expressed in fetal lung throughout all studied gestational ages. MCT1 and MCT4 are predominantly expressed in the plasma membrane of bronchiolar and alveolar epithelial cells from all studied gestational stages, while immunoreaction in mesenchymal cells is observed for MCT1 at $17.5 \mathrm{dpc}$ and $19.5 \mathrm{dpc}$ and for MCT4 at 15.5, 17.5 and $19.5 \mathrm{dpc}$. Western blot analysis for MCT1 and MCT4 reveals bands around $50 \mathrm{kDa}$ and $43 \mathrm{KDa}$, respectively, consistent with the molecular weight described for these proteins (Fig. 2B). Thus, we confirm that both MCT1 and MCT4 are expressed throughout all gestational stages in rat fetal lung.

\section{Effect of MCT inhibition on rat lung morphogenesis}

We aimed to understand the contribution of MCTs to lung morphogenesis. For that we cultured rat fetal lung explants and MCT activity was inhibited with CHC, a classical MCT inhibitor.

For this study, 30 lung explants were cultured with two doses of $\mathrm{CHC}(0.5 \mathrm{mM}$ and $1 \mathrm{mM}$ ). Representative pictures of fetal lung explants are illustrated in figure $3 \mathrm{~A}$. Comparing 
fetal lung explants treated with CHC at D4 with control (DMSO) it appears that CHC has an inhibitory effect in both doses studied (Fig. 3B). Morphometric analyses show that $1 \mathrm{mM}$ $\mathrm{CHC}$ has a significant inhibitory effect on branching morphogenesis (Fig. 3Biii) $(\mathrm{p}<0.05)$. There was a decrease of the epithelial perimeter (Fig.3Bi/ii) and area (Fig.3Biv/v) of lung explants, in a dose dependent way, being significant for $1 \mathrm{mM} \mathrm{CHC}(\mathrm{p}<0.05)$.

\section{Discussion}

Hypoxia is a normal component of embryo development [31], and it seems to be one of the most important extracellular factors for lung morphogenesis $[17,18]$ which is a complex process [18]. In most mammalian systems, the cellular responses to oxygen alterations are mediated through the hypoxia-inducible factor (HIF) family of transcriptional regulators $[32,33]$. HIF complex is a heterodimer composed of one of three $\alpha$-subunits (HIF- $1 \alpha$, HIF$2 \alpha$, or HIF-3 $\alpha$ ) and a $\beta$-subunit (ARNT; also known as aryl hydrocarbon receptor nuclear translocator). In fetal lung, HIF mRNA and protein levels are quite high [26, 32, 34], being the HIF-1 $\alpha$ protein localized predominantly in the branching epithelium [34]. These authors showed that induction of HIF-1 protein in fetal lung buds was sufficient to induce lung development even under non-hypoxic conditions, whereas its depletion, using antisense oligonucleotides, reduced lung branching morphogenesis and vascularization [35]. The enhancement of lung epithelial branching morphogenesis by low oxygen has also been reported by Gebb and Jones [36].

Hypoxic environment favours anaerobic glycolysis. This metabolic phenotype is characterized by the switch of cell metabolism from oxidative phosphorylation to glycolysis, being pyruvate metabolized to lactate [37] which is then exported by MCTs to avoid a decrease in pHi.

Although the contribution of MCTs to the glycolytic and acidic phenotype of tumours is well studied [6], their contribution to the embryo development is still not clear. Several studies have already suggested that embryos, as proliferating cells, express a metabolic phenotype similar to cancer cells [37]. We showed that lung cells develop in an intermittent hypoxia environment by the expression of the hypoxia marker CAIX. These results are in agreement with previous studies that report expression of HIF during lung development $[32,34]$.

In order to adapt to intermittent hypoxia, cells need to create conditions to support embryogenesis, increasing the expression of genes related to the metabolic phenotype such as GLUT1 and MCT1/4 and CD147. It has also been described that HIF-1 $\alpha$ up-regulates both GLUT1 and MCT4 [38]. Thus, with this study we show for the first time the expression pattern of MCT1/4 and their chaperone CD147 during human lung embryo development. We demonstrate that in normal fetal lung, MCT1/4 isoforms are expressed throughout the lung development phases suggesting that these molecules are important for lung morphogenesis. MCT1 was seen in the pseudoglandular phase in all cellular types, becoming absent in mesenchymal cells along gestation, being detected only in alveolar epithelium cells in later gestation, while MCT4 was predominantly found to be expressed throughout the various phases in bronchial epithelium. These results suggest that MCT1 might have a role in alveolar epithelium and MCT4 is more important in bronchial epithelium. CD147 expression was similar to both MCT isoforms, however from week 18 to week 22 it is not detected by immunohistochemistry in alveolar epithelium where MCT1 is present. This finding was unexpected since it has been described that MCT1/4 require this chaperone to be trafficked to the plasma membrane and perform their activity $[11,12]$, which may suggest the existence of another chaperone. We also analysed the expression of GLUT1, a glucose transporter widely distributed in fetal tissues that has been shown to be overexpress in tissues with high glycolytic rates, as well as CAIX. GLUT1 expression was similar to MCT4 through gestation but absent in the last phase of lung development (saccular/alveolar), while CAIX 
expression varies along gestation, starting to be expressed only in bronchial epithelium and being present in all epithelial cells in later phases. These results suggest that lungs develop in a hypoxic environment and that cells have the machinery to adapt to diferent levels of $\mathrm{O}_{2}$. Our results show that the glycolytic metabolic phenotype is more pronounced in the early phases by the expression of glucose transporter (GLUT1) and lactate exporters (MCT1/4). This phenotype continues to be detected in the bronchial epithelium during the canalicular phase. However, in the late stage of gestation this phenotype is attenuated. Besides the expression of the hypoxia marker CAIX in most of the cells, expression of MCT4 is restricted to bronchial epithelium and GLUT1 is absent. At this level, lung tissue is close to complete maturation and the rates of proliferation decrease. The expression of CAIX at this time was not expected. Liao et al showed that CAIX was highly expressed during the second trimester of gestation but decreased after week 30 [39].

Several studies have already demonstrated the expression of MCTs during embryo development specifically during pre-implantation development of the human and mouse [7, $23,24]$. Some studies showed that since the development of the zygote until the stage of 2 cells, there is an absolute requirement for pyruvate [40] and lactate to support the development of the embryo in stages of 4 and 8 cells $[23,41]$. However, from stage 8 it has been observed that glucose consumption increases, being the main carbon and energy source to the embryo [42]. The importance of MCTs has been already described in other organs during rat embryo development [21, 25, 43]. Han et al. [21] detected MCT4 and the chaperone CD147 by immunohistochemistry during early cardiomyocyte differentiation and another study describes the immunoexpression of MCT1 and MCT2 in various cell populations of the rat forebrain, from the late embryonic period to the early post-natal period [25].

In order to understand the influence of MCT inhibition in lung morphogenesis, studies using fetal lung explant models cultured under CHC exposure were performed. First, in rat tissues we confirmed the expression of both MCT1 and MCT4 during rat lung development. We showed that MCT1 and MCT4 were expressed in primitive epithelial cells as early as the pseudoglandular stage and were also both detected in the bronchial and alveolar epithelium cells in the remaining phases. The immunohistochemical results were corroborated by Western blot. For in vitro studies, two different doses of CHC, MCT inhibitor, were added to lung explants and it was observed that lung branching was inhibited with increasing concentrations of CHC. Morphometric analysis was performed to determine the number of peripheral airway buds, epithelial perimeter and total area. We observed a significant effect for $1 \mathrm{mM}$ CHC in the number of peripheral airway buds. Although CHC is considered as a classical inhibitor of MCT activity, it is also described to inhibit the mitochondrial pyruvate transporter [44, 45]. This was described by Halestrap and co workers in 1974 [45], however in this study they use isolated mitochondria. On one hand, there are no studies reporting the entry of CHC into cells and, on the other hand, there is evidence showing that CHC acts only outside the cell [45-48]. Thus, we believe that effect of CHC in our model is mediated by inhibition of MCT activity at the plasma membrane. However, further experiments should be performed to clarify the true contribution of MCT inhibition in lung branching. Experiments with the specific inhibitor of MCT1/2 from AstraZeneca [49] or even RNA interference approaches could be used.

Although the role of MCTs in lung morphogenesis is still far from being well understood, with this study we showed that CHC disrupts lung branching, suggesting the dependence of these transporters for lung development. Embryonic developmental is strongly influenced by oxygen availability in the environment [20] however, the explants cultures were performed in normoxia conditions, what could not reflect the physiologic conditions in vivo. From our results, we hypothesize that lung embryonic cells are able to survive in hypoxia environments adopting a metabolic phenotype to supply energy and that MCTs/hypoxia may have different and specific functions in epithelial differentiation and vascular morphogenesis. Nevertheless, further studies are needed to better characterize this metabolic phenotype during lung differentiation. 
Granja et al.: CHC Affects Lung Branching

\section{Acknowledgements}

This work was supported by the Life and Health Sciences Research Institute, University of Minho, Portugal, and Fundação para a Ciência e Tecnologia (SFRH/BD/33503/2010 to S.G., SFRH/BD/ 87139/2012 to F.M.S. and SFRH/BD/51997/2012 to V.M.G.)

\section{References}

1 Tuder RM, Davis LA, Graham BB: Targeting energetic metabolism: a new frontier in the pathogenesis and treatment of pulmonary hypertension. Am J Respir Crit Care Med 2012;185:260-266.

- Jezek P, Plecitá-Hlavatá L, Smolková K, Rossignol R: Distinctions and similarities of cell bioenergetics and the role of mitochondria in hypoxia, cancer, and embryonic development. Int J Biochem Cell Biol 2010;42:604-622.

3 Harding EA, Gibb CA, Johnson MH, Cook DI, Day ML: Developmental Changes in the Management of Acid Loads During Preimplantation Mouse Development. Biol Reprod 2002;67:1419-1429.

4 Halestrap AP: The monocarboxylate transporter family-Structure and functional characterization. IUBMB life 2012;64:1-9.

5 Merezhinskaya N, Fishbein WN: Review Monocarboxylate transporters: Past, present, and future. Histol Histopathol 2009;24:243-264.

6 Pinheiro C, Longatto-Filho A, Azevedo-Silva J, Casal M, Schmitt FC, Baltazar F: Role of monocarboxylate transporters in human cancers: state of the art. J Bioenerg Biomembr 2012;44:127-139.

7 Hérubel F, Mouatassim S El, Guérin P, Frydman R, Ménézo Y: Genetic expression of monocarboxylate transporters during human and murine oocyte maturation and early embryonic development. Zygote 2002;10:175-181.

8 Dimmer K, Lang F, Deitmer JW, Bro S: The low afinity of monocarboxylate transporter MCT4 is adapted to the export of lactate in highly glycolytic cells. Biochem J 2000;357:219-227.

-9 Halestrap AP, Price NT: The proton-linked monocarboxylate transporter (MCT) family: structure, function and regulation. Biochem J 1999;343:281-299.

10 Fox JEM, Meredith D, Halestrap AP: Characterisation of human monocarboxylate transporter 4 substantiates its role in lactic acid efflux from skeletal muscle. J Physiol 2000;529:285-293.

11 Wilson MC, Meredith D, Fox JEM, Manoharan C, Davies AJ, Halestrap AP: Basigin (CD147) Is the Target for Organomercurial Inhibition of Monocarboxylate Transporter Isoforms 1 and 4. J Biol Chem 2005;280:27213-27221.

12 Kirk P, Wilson MC, Heddle C, Brown MH, Barclay AN, Halestrap AP: CD147 is tightly associated with lactate transporters MCT1 and MCT4 and facilitates their cell surface expression. EMBO J 2000;19:3896-3904.

13 Gallagher SM, Castorino JJ, Wang D, Philp NJ: Monocarboxylate Transporter 4 Regulates Maturation and Trafficking of CD147 to the Plasma Membrane in the Metastatic Breast Cancer Cell Line MDA-MB-231. Cancer Res 2007;67:4182-4189.

14 Jauniaux E, Watson A, Burton G: Evaluation of respiratory gases and acid-base gradients in human fetal fluids and uteroplacental tissue between 7 and 16 weeks' gestation. Am J Obstet Gynecol 2001;184:9981003.

15 Groenman FA, Rutter M, Wang J, Caniggia I, Tibboel D, Post M: Effect of chemical stabilizers of hypoxiainducible factors on early lung development. Am J Physiol Lung Cell Mol Physiol 2007;293:L557-567.

- 16 Lee YM, Jeong C, Koo S, Son MJIN, Song HS, Bae S, Raleigh JA, Chung HJ, Yoo MA: Determination of Hypoxic Region by Hypoxia Marker in Developing Mouse Embryos In Vivo : A Possible Signal for Vessel Development. Dev Dyn 2001;186:175-186.

17 Troug W, Svojanovsky S, Soares MJ: Chronic Hypoxia and Rat Lung Development: Analysis by Morphometry and Directed Microarray. Pediatr Res 2008;64:56-62.

18 Saini Y, Harkema JR, Lapres JJ: Hif1 $\alpha$ is essential for normal intrauterine diferentiation of alveolar epithelium and surfactant production in the newborn lung of mice. J Biol Chem 2008;48:33650-657.

19 Gebb SA, Fox K, Vaughn J, McKean D, Jones PL: Fetal oxygen tension promotes tenascin-C-dependent lung branching morphogenesis. Dev Dyn 2005;234:1-10. 


\section{Cellular Physiology $\quad$ Cell Physiol Biochem 2013;32:1845-1856 and Biochemistry \\ Granja et al.: CHC Affects Lung Branching}

20 Chen EY, Fujinaga M, Giaccia AJ: Hypoxic Microenvironment Within an Embryo Induces Apoptosis and Is Essential for Proper Morphological Development. Teratology 1999;60:215-225.

21 Han M, Trotta P, Coleman C, Linask KK: MCT-4, A511/Basigin and EF5 Expression Patterns During Early Chick Cardiomyogenesis Indicate Cardiac Cell Differentiation Occurs in a Hypoxic Environment. Dev Dyn 2005;235:124-131.

-22 Jansen S, Pantaleon M, Kaye PL: Characterization and regulation of monocarboxylate cotransporters Slc16a7 and Slc16a3 in preimplantation mouse embryos. Biol Reprod 2008;79:84-92.

-23 Harding EA, Day ML, Gibb C a, Johnson MH, Cook DI: The activity of the $\mathrm{H}^{+}$-monocarboxylate cotransporter during pre-implantation development in the mouse. Pflügers Archiv 1999;438:397-404.

-24 Jansen S, Pantaleon M, Esmaeilpour T, Kaye PL: Glucose affects monocarboxylate cotransporter (MCT) 1 expression during mouse preimplantation development. Reproduction 2006;131:469-479.

-25 Baud O, Fayol L, Gressens P, Pellerin L, Magistretti P, Evrard P, Verney C: Perinatal and early postnatal changes in the expression of monocarboxylate transporters MCT1 and MCT2 in the rat forebrain. J Comp Neurol 2003;465:445-454.

26 Semenza GL: HIF-1: mediator of physiological and pathophysiological responses to hypoxia. J Appl Physiol 2000;88:1474-1480.

27 Scott CL, Walker DJ, Cwiklinski E, Tait C, Tee a R, Land SC: Control of HIF-1\{alpha\} and vascular signaling in fetal lung involves cross talk between mTORC1 and the FGF-10/FGFR2b/Spry2 airway branching periodicity clock. Am J Physiol Lung Cell Mol Physiol 2010;299:L455-471. Webster WS, Abela D: The Effect of Hypoxia in Development. Birth Defects Res 2007;81:215-228. Miranda-Gonçalves V, Honavar M, Pinheiro C, Martinho O, Pires MM, Pinheiro C, Cordeiro M, Bebiano G, Costa P, Palmeirim I, Reis RM, Baltazar F: Monocarboxylate transporters (MCTs) in gliomas: expression and exploitation as therapeutic targets. Neuro Oncol 2013;15:172-188.

-30 Pinheiro C, Sousa B, Albergaria A, Paredes J, Dufloth R, Vieira D, Schmitt F, Baltazar F: GLUT1 and CAIX expression profiles in breast cancer correlate with adverse prognostic factors and MCT1 overexpression. Histol Histopathol 2011;26:1279-1286.

31 Simon MC, Keith B: The role of oxygen availability in embryonic development and stem cell function. Nat Rev Mol Cell Biol 2008;9:285-296.

-32 Shimoda LA, Semenza GL: HIF and the lung: role of hypoxia-inducible factors in pulmonary development and disease. Am J Respir Crit Care Med 2011;183:152-156.

-33 Semenza GL, Hif- H: HIF-1 and mechanisms of hypoxia sensing. Curr Opin Cell Biol 2001;13:167-171.

34 Groenman F, Rutter M, Caniggia I, Tibboel D, Post M: Hypoxia-inducible factors in the first trimester human lung. J Histochem Cytochem 2007;55:355-363.

-35 Van Tuyl M, Liu J, Wang J, Kuliszewski M, Tibboel D, Post M: Role of oxygen and vascular development in epithelial branching morphogenesis of the developing mouse lung. Am J Physiol Lung Cell Mol Physiol 2005;288:L167-178. Gebb S, Jones P: Hypoxia and lung branching morphogenesis. Adv Exp Med Biol 2003;543:117-125. Krisher RL, Prather RS: A role for the Warburg effect in preimplantation embryo development: metabolic modification to support rapid cell proliferation. Mol Reprod Dev 2012;79:311-320.

38 Ullah MS, Davies AJ, Halestrap AP: The Plasma Membrane Lactate Transporter MCT4, but Not MCT1, Is Upregulated by Hypoxia through a HIF-1-dependent Mechanism. J Biol Chem 2006;281:9030-9037.

39 Liao SY, Lerman MI, Stanbridge EJ: Expression of transmembrane carbonic anhydrases, CAIX and CAXII, in human development. BMC Dev Biol 2009;9:22

40 Harris SE, Leese HJ, Gosden RG, Picton HM: Pyruvate and oxygen consumption throughout the growth and development of murine oocytes. Mol Reprod Dev 2009;76:231-238.

41 Lane M, Gardner DK: Lactate Regulates Pyruvate Uptake and Metabolism in the Preimplantation Mouse Embryo. Biol Reprod 2000;62:16-22.

42 Butcher L, Coates A, Martin KL, Rutherford AJ, Leese HJ: Metabolism of Pyruvate by the Early Human Embryo. Biol Reprod 1998;1056:1054-1056.

43 Clamp MF, Ochrietor JD, Moroz TP, Linser PJ: Developmental analyses of 5A11/Basigin, 5A11/Basigin-2 and their putative binding partner MCT1 in the mouse eye. Exp Eye Res 2004;78:777-789.

-44 Halestrap AP: The mitochondrial pyruvate carrier. Kinetics and specificity for substrates and inhibitors. Biochem J 1975;148:85-96. 
45 Halestrap AP, Denton RM: Specific inhibition of pyruvate transport in rat liver mitochondria and human erythrocytes by alpha-cyano-4-hydroxycinnamate. Biochem J 1974;138:313-316.

-46 Gäreskog M, Wentzel P: N-Acetylcysteine and alpha-cyano-4-hydroxycinnamic acid alter protein kinase C (PKC)-delta and PKC-zeta and diminish dysmorphogenesis in rat embryos cultured with high glucose in vitro. J Endocrinol 2007;192:207-214.

47 Colen CB, Seraji-Bozorgzad N, Marples B, Galloway MP, Sloan AE, Mathupala SP: Metabolic remodeling of malignant gliomas for enhanced sensitization during radiotherapy: an in vitro study. Neurosurgery 2006;59:1313-1323; discussion 1323-1324.

-48 Colen CB, Shen Y, Ghoddoussi F, Yu P, Francis TB, Koch BJ, Monterey MD, Galloway MP, Sloan AE, Mathupala SP: Metabolic targeting of lactate efflux by malignant glioma inhibits invasiveness and induces necrosis: an in vivo study. Neoplasia 2011;13:620-632.

49 Ovens MJ, Davies AJ, Wilson MC, Murray CM, Halestrap AP: AR-C155858 is a potent inhibitor of monocarboxylate transporters MCT1 and MCT2 that binds to an intracellular site involving transmembrane helices 7-10. Biochem J 2010;425:523-530. 